\author{
Y. Mitamura - A. Okumura $\cdot$ C. Harada $\cdot$ \\ K. Namekata $\cdot$ K. Nakamura $\cdot$ A. Tashimo $\cdot$ \\ K. Ohtsuka - T. Harada
}

\title{
Activator protein-1 in epiretinal membranes of patients with proliferative diabetic retinopathy
}

Received: 19 July 2005 / Accepted: 7 September 2005 / Published online: 9 December 2005

(C) Springer-Verlag 2005

\begin{abstract}
Abbreviation AP-1: activator protein-1
To the Editor: Angiogenesis is the most important event in proliferative diabetic retinopathy. Previous studies indicated that activator protein-1 (AP-1) is involved in angiogenesis $[1,2]$. AP-1 is a transcription factor and a homodimer or heterodimer mainly composed of Jun-Jun and Jun-Fos. The gene encoding Jun was originally identified in avian sarcoma virus 17, and the abbreviation 'Jun' is derived from the Japanese 'ju-nana', which means 17. cJun (encoded by $J U N$ ) is a member of the Jun family and is a principal component of AP-1. The aim of the present study was to assess the potential role of AP-1 in the pathogenesis of proliferative diabetic retinopathy. For this purpose, we examined the expression of $J U N$ mRNA in epiretinal membranes secondary to proliferative diabetic retinopathy. Because perivascular glial cells are essential for the regulation of retinal angiogenesis [3], we also examined levels of phosphorylated c-Jun in glial cells from epiretinal membranes.

Epiretinal membranes were surgically removed from consecutive patients with proliferative diabetic retinopathy (23 type 2 diabetic patients) or idiopathic epiretinal
\end{abstract}

Y. Mitamura $(\bowtie) \cdot$ A. Tashimo $\cdot$ K. Ohtsuka

Department of Ophthalmology,

School of Medicine,

Sapporo Medical University,

S-1, W-16, Chuo-ku,

Sapporo, 060-8543 Japan

e-mail: ymita@sapmed.ac.jp

Tel.: +81-11-611-2111

Fax: $+81-11-613-6575$

A. Okumura - C. Harada · K. Namekata .

K. Nakamura $\cdot$ T. Harada

Department of Molecular Neurobiology,

Tokyo Metropolitan Institute for Neuroscience,

Fuchu,

Tokyo, Japan membranes (controls, 16 non-diabetic patients) during pars plana vitrectomy. The epiretinal membrane in proliferative diabetic retinopathy is mainly composed of neovascular vessels. On the other hand, idiopathic epiretinal membrane is a non-angiogenic fibroglial membrane that is not associated with diabetes mellitus. Samples derived from 19 of the 23 patients with proliferative diabetic retinopathy and the 16 control patients were processed for RT-PCR analysis. The remaining samples from the diabetic patients were processed for immunohistochemistry. Informed consent was obtained from each patient. The present investigations were carried out in accordance with the principles of the Declaration of Helsinki as revised in 2000.

RT-PCR analysis was performed as previously described $[4,5]$. The primer sequences used to target the genes in PCR were as follows: $J U N$ : 5'-GCA TGA GGA ACC GCA TCG CTG CCT CCA AG-3' and 5'-GAC CAA GTC CTT CCC ACT CGT GCA CAC TG-3'; and GAPDH: 5'-ACC ACA GTC CAT GCC ATC AC-3' and 5'-TCC ACC ACC CTG TTG CTG TA-3'. Reactions were conducted under the following conditions: precycling at $94^{\circ} \mathrm{C}$ for $3 \mathrm{~min}$, then 35 cycles consisting of denaturation at $94^{\circ} \mathrm{C}$ for $30 \mathrm{~s}$, annealing at either $62^{\circ} \mathrm{C}(J U N)$ or $55^{\circ} \mathrm{C}(G A P D H)$ for $30 \mathrm{~s}$ and polymerisation at $72^{\circ} \mathrm{C}$ for $1 \mathrm{~min}$. The expected sizes of the amplified cDNA fragments of $J U N$ and GAPDH were 409 and $452 \mathrm{bp}$, respectively.

Immunohistochemical analysis was performed as previously described $[4,5]$. The following antibodies were used to detect antigens: a goat polyclonal antibody against phosphorylated c-Jun (1:200 dilution; Santa Cruz Biotechnology, Santa Cruz, CA, USA) and a mouse monoclonal antibody against vimentin (1:500 dilution; Nichirei, Tokyo, Japan). The antibodies were detected with $\mathrm{Cy} 3$-conjugated donkey anti-goat $\operatorname{IgG}(1: 1,000$ dilution; Jackson Immunoresearch, West Grove, PA, USA) and Cy2-conjugated donkey anti-mouse IgG (1:200 dilution; Jackson Immunoresearch), respectively. To visualise all nuclei, the 
sections were counterstained with Hoechst 33342 (bisbenzimide; 1:1,000 dilution; Molecular Probes, Eugene, OR, USA).

Data were analysed using Fisher's exact test. A $p$ value of less than 0.05 was considered statistically significant.

In RT-PCR analysis, $J U N$ mRNA was detected in all 19 patients with proliferative diabetic retinopathy (100\%), but in only seven of the 16 control patients (44\%) (Fig. 1a). There was a significant difference between the two groups $(p=0.0002)$.

Immunohistochemical analysis was performed to identify phosphorylated c-Jun in epiretinal membranes from patients with proliferative diabetic retinopathy. Phosphorylated c-Jun was detected in all four of the diabetic patients examined (Fig. 1b). We next examined whether phosphorylated c-Jun can be found in glial cells. Epiretinal membranes contained a large area composed of glial cells (Fig. 1c), and many cells in this area were double-labelled for phosphorylated c-Jun (Fig. 1e). Similar results were
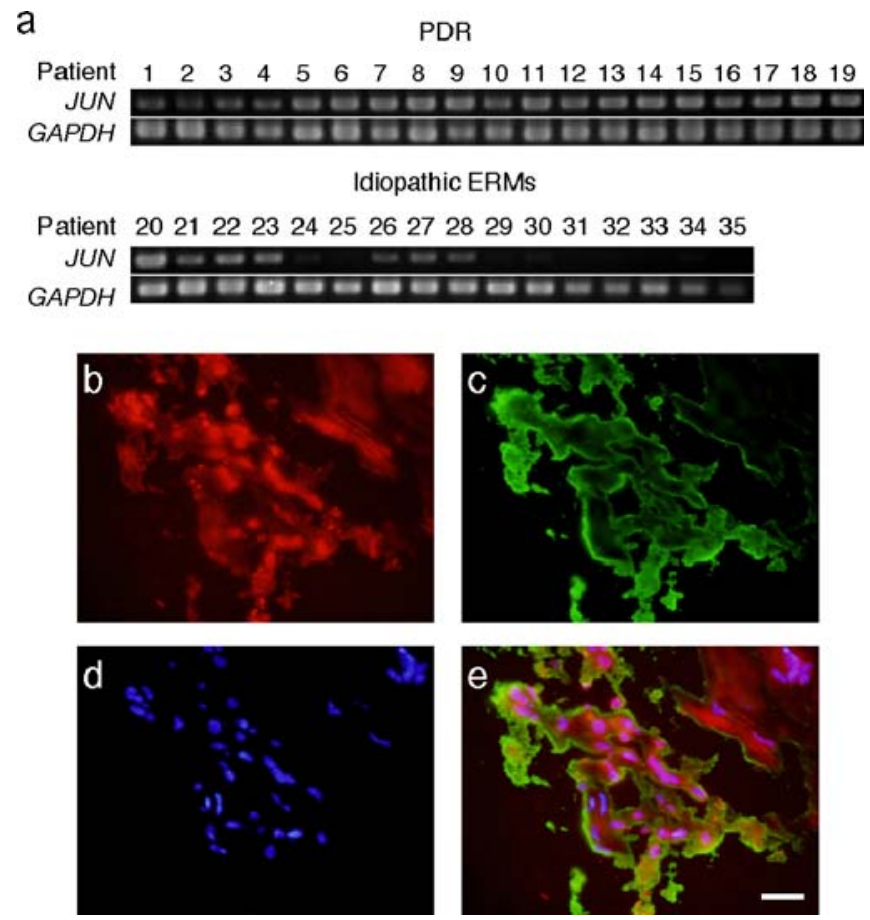

Fig. 1 a RT-PCR analysis of c-Jun in epiretinal membranes derived from patients with proliferative diabetic retinopathy (PDR; patients 1-19) and idiopathic epiretinal membranes (idiopathic ERMs; patients 20-35). After 35 cycles, $15 \mu \mathrm{l}$ of each sample was electrophoresed through a $2 \%$ Tris-acetate-EDTA agarose gel and the fractionated products were stained with ethidium bromide. Note the high level of expression of $J U N$ mRNA in epiretinal membranes derived from patients with proliferative diabetic retinopathy. b-e Detection of phosphorylated c-Jun (b) and vimentin (c) in epiretinal membrane derived from a 51-year-old proliferative diabetic retinopathy patient with a 12-year history of diabetes. d Nuclear staining of the same section. e Triple-labelling demonstrates the presence of phosphorylated c-Jun in glial cells. Bar: $50 \mu \mathrm{m}$ obtained in experiments using various glial cell markers, such as glutamine synthetase (data not shown).

In the present study, we found that $J U N$ mRNA is highly expressed in epiretinal membranes from patients with proliferative diabetic retinopathy. Binding sites for AP-1 are ubiquitous among gene promoters of various angiogenesis-related cytokines, such as IL-8, vascular endothelial growth factor and basic fibroblast growth factor [6]. These cytokines can be detected in vitreous fluid and epiretinal membrane samples derived from patients with proliferative diabetic retinopathy [7]. In particular, vascular endothelial growth factor is considered to be the most attractive candidate for proliferative diabetic retinopathy. Okamoto et al. [1] reported that AGEs stimulate the growth of and tube formation by microvascular endothelial cells and upregulate the transcription of the gene encoding vascular endothelial growth factor through AP-1. In addition, the overexpression of a transfected gene encoding c-Fos induces severe angiogenesis in the rat brain [2]. Taken together, our results suggest that AP-1 plays a role in the angiogenesis of proliferative diabetic retinopathy by regulating the promoter activity and expression of genes encoding angiogenesis-related cytokines. However, we cannot clarify whether the increased production of AP-1 is the cause or the effect of proliferative diabetic retinopathy in this study. There is a possibility that the increased production of AP-1 may be secondary to the increased levels of inflammatory cytokines observed in proliferative diabetic retinopathy [8]. Further investigations are needed to understand the precise role of $\mathrm{AP}-1$ in proliferative diabetic retinopathy.

We have also demonstrated that phosphorylated c-Jun is widely found in the glial component of epiretinal membranes of patients with proliferative diabetic retinopathy. The glial cell is one of the main components of the epiretinal membrane in proliferative diabetic retinopathy, and can secrete angiogenesis-related cytokines, such as vascular endothelial growth factor and basic fibroblast growth factor $[5,7,9]$. The functional role of glial cells in endothelial cell proliferation has been demonstrated using an astrocyte/endothelial co-culture as an in vitro model of angiogenesis [3]. In addition, there is strong immunostaining of vascular endothelial growth factor in glial cells from retinas of patients with diabetes mellitus before the development of proliferative diabetic retinopathy [9]. These results provide evidence that glial cells are the initial cellular sites of molecular events that may lead to retinal neovascularisation. Thus, the inhibition of AP-1 activation in glial cells may modify interactions between glial cells and vascular endothelial cells and prevent the angiogenesis of proliferative diabetic retinopathy.

Acknowledgements Supported by the Ministry of Education, Culture, Sports, Science and Technology of Japan (Y. Mitamura and T. Harada); the Japan Society for the Promotion of Science for Young Scientists (C. Harada); and the Japan Diabetes Foundation (T. Harada). 


\section{References}

1. Okamoto T, Yamagishi S, Inagaki Y et al (2002) Angiogenesis induced by advanced glycation end products and its prevention by cerivastatin. FASEB J 6:1928-1930

2. Asakuno K, Isono M, Wakabayashi $Y$ et al (1995) The exogenous control of transfected c-fos gene expression and angiogenesis in cells implanted into the rat brain. Brain Res $702: 23-31$

3. Laterra J, Goldstein GW (1991) Astroglial-induced in vitro angiogenesis: requirements for RNA and protein synthesis. J Neurochem 57:1231-1239

4. Harada T, Harada C, Mitamura Y et al (2002) Neurotrophic factor receptors in epiretinal membranes after human diabetic retinopathy. Diabetes Care 25:1060-1065

5. Mitamura Y, Harada T, Harada C et al (2003) NF- $к B$ in epiretinal membranes after human diabetic retinopathy. Diabetologia 46:699-703

6. Yoshida S, Ono M, Shono T et al (1997) Involvement of interleukin-8, vascular endothelial growth factor, and basic fibroblast growth factor in tumor necrosis factor alpha-dependent angiogenesis. Mol Cell Biol 17:4015-4023

7. Mitamura Y, Harada C, Harada T (2005) Role of cytokines and trophic factors in the pathogenesis of diabetic retinopathy. Curr Diab Rev 1:73-81

8. Kyriakis JM (1999) Activation of the AP-1 transcription factor by inflammatory cytokines of the TNF family. Gene Expr 7:217-231

9. Amin RH, Frank RN, Kennedy A, Eliott D, Puklin JE, Abrams GW (1997) Vascular endothelial growth factor is present in glial cells of the retina and optic nerve of human subjects with nonproliferative diabetic retinopathy. Invest Ophthalmol Vis Sci 38:36-47 